\title{
Impacts of Trapezoidal Fin of 20-nm Double-Gate FinFET on the Electrical Characteristics of Circuits
}

\author{
Myunghwan Ryu ${ }^{1}$ and Youngmin $\mathrm{Kim}^{2}$
}

\begin{abstract}
In this study, we analyze the impacts of the trapezoidal fin shape of a double-gate FinFET on the electrical characteristics of circuits. The trapezoidal nature of a fin body is generated by varying the angle of the sidewall of the FinFET. A technology computeraided-design (TCAD) simulation shows that the onstate current increases, and the capacitance becomes larger, as the bottom fin width increases. Several circuit performance metrics for both digital and analog circuits, such as the fan-out 4 (FO4) delay, ring oscillator (RO) frequency, and cut-off frequency, are evaluated with mixed-mode simulations using the 3D TCAD tool. The trapezoidal nature of the FinFET results in different effects on the driving current and gate capacitance. As a result, the propagation delay of an inverter decreases as the angle increases because of the higher on-current, and the FO4 speed and RO frequency increase as the angle increases but decrease for wider angles because of the higher impact on the capacitance rather than the driving strength. Finally, the simulation reveals that the trapezoidal angle range from $10^{\circ}$ to $20^{\circ}$ is a good tradeoff between larger on-current and higher capacitance for an optimum trapezoidal FinFET shape.
\end{abstract}

Index Terms-FinFET, trapezoidal fin shape, multigate transistor, double gate, SOI FinFET, mixed-mode 3D TCAD

\footnotetext{
Manuscript received Mar. 28, 2015; accepted Jul. 26, 2015

${ }^{1}$ School of Electrical and Computer Engineering, Ulsan National Institute of Science and Technology (UNIST).

${ }^{2}$ School of Computer Engineering, Kwangwoon University

E-mail : youngmin@kw.ac.kr
}

\section{INTRODUCTION}

As CMOS technologies reach the deep submicron (DSM) regime through aggressive scaling, integrated circuits (ICs) have encountered scaling impediments. Short-channel effects (SCEs) such as subthreshold swing degradation, source/drain leakage current problems, larger drain-induced barrier lowering (DIBL), $V_{\text {th }}$ roll-off, and $V_{\text {th }}$ mismatch caused by random dopant fluctuations lead to dramatic change in the use of conventional planar devices for IC designs [1]. The classical solution for the reduced gate controllability issues in DSM has been to increase the gate capacitance by reducing the oxide thickness in proportion to the channel length. However, this approach is no longer effective to meet the device requirements (e.g., leakage) beyond a 32-nm technology. To address this issue, multi-gate structures are introduced to increase the gate controllability [2]. Among the several structures of multi-gate transistors, the FinFET is one of the promising alternatives to conventional planar devices because of its better immunity to SCEs, higher current drive strength, and improved subthreshold swing [3]. In addition, the FinFET is the most promising alternative because the fabrication of FinFETs is very similar to that of conventional MOSFETs compared to other multi-gate technologies [4].

Recently, the leading IC manufacturer introduced the first FinFET-based chip [5]. Additionally, it was revealed that the microscopic cross sections of the fin body of the transistor in the processor actually have a trapezoidal shape, as shown in Fig. 1 [6-8]. Although a FinFET is believed to have uniform thickness across the height of a fin, the trapezoidal cross section has been found to be markedly different from the idealized rectangular fin 


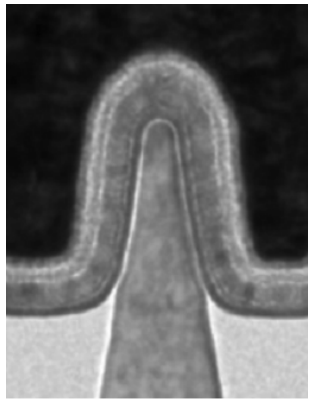

(a)

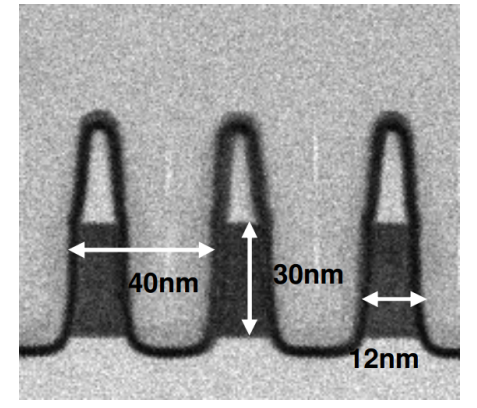

(b)
Fig. 1. Cross-sectional TEM images of (a) a FinFET channel [7], (b) multi-finger FinFET arrays [8] under the gate along the fin-width direction.

body in many studies [9]. It is not clearly known whether the nonvertical cross section of the fins are manufacturing artifacts or are deliberately engineered to provide useful benefits in the chip. In [7], the authors claimed that obtaining a uniform thickness across the entire height of the fin is extremely difficult in the current process technology. Thus, its real geometry has an angle of inclination along the fin height. Whether intentional or due to manufacturing imperfections, the real trapezoidal cross section requires appropriate models to predict electrical behaviors.

The impacts of the angle variation along the height of the fin have been investigated by TCAD simulations in previous works [10-12]. In [10], the impact of a nonrectangular fin cross section on the electrical characteristics of a FinFET was characterized. However, the paper analyzed the impact on the device level only and did not address the impact of angle variation on the circuit level. In [11], although the electron and current density profiles as a function of inclination angle for various body doping levels are investigated by TCAD simulations, the authors did not take circuit-level performance into account. Another paper [12] has claimed that a triangular shape, an extreme case of a trapezoidal shape, of a FinFET provides advantages in performance and energy savings, resulting in the ring oscillator operating faster than a FinFET with rectangular fins. However, only two cases (rectangular and triangular case ( $8^{\circ}$ inclination angle)) were analyzed in their work without analyzing other trapezoidal angle. Very few studies have been conducted to investigate the optimum trapezoidal angle for the dynamic performance of the circuits for various angle.
In this study, we analyze the impacts of a trapezoidal fin shape on the electrical characteristics of FinFETbased circuits. FinFET structures with angular variations of the sidewalls are exploited in 3D device simulations, and several circuit performance metrics such as the fanout 4 (FO4) and ring oscillator (RO) delays and cut-off frequency are evaluated with a mixed-mode method (i.e., transient and AC analysis by TCAD) in Sentaurus Device [13] simulations for dynamic performance analysis. The main contributions of our work are summarized as follows:

- We exploit 3D TCAD simulations to analyze the device level effects by various trapezoidal angle variations.

- We extend the device level analysis to the circuit level performance metric by using of mixed-mode simulations.

- We propose the optimum trapezoidal angle in circuit performance through the combined analysis.

The rest of this paper is organized as follows. Section II presents the base 3D structure and explains the electrical properties of the trapezoidal FinFET. Impact of angular variation is explained in Section III. Simulation results of the impact on the circuit performance are discussed in Section IV, followed by conclusions in Section V.

\section{TrapezoidAL FinfeT}

FinFETs with a rectangular channel shape and a trapezoidal (nonvertical) shape used in this paper are shown in Fig. 2. The cross sections through the fin channel (perpendicular to the flow of the current) are shown in (b) and (c) for the rectangular and trapezoidal fins, respectively. The rectangular channel shape of the FinFET is used as the base structure. The Sentaurus Structure Editor [13] is used to construct the 3D FinFET structures. As can be observed, the FinFET is created on buried oxide (BOX). The parameters used in the TCAD simulation are shown in Table 1. A high-k metal-gate (HKMG) process is used to construct the gate region. We implement a thicker top oxide to form double-gate structures. The widths of the upper and lower parts of the fin channel are WFin,top and WFin,bot, respectively. Although the upper level of the fin is fixed to a constant 
Table 1. Physical parameters used in the device simulation

\begin{tabular}{|c|c|}
\hline Parameters & Value \\
\hline Channel length $\left(L_{\mathrm{g}}\right)$ & $23 \mathrm{~nm}$ \\
\hline Height of fin $\left(H_{\text {fin }}\right)$ & $28 \mathrm{~nm}$ \\
\hline Width of top fin $($ WFin,top $)$ & $15 \mathrm{~nm}$ \\
\hline Gate oxide thickness for top $\left(T_{\text {ox,top }}\right)$ & $14 \mathrm{~nm}$ \\
\hline Gate oxide thickness for side $\left(T_{\text {ox,side }}\right)$ & $1.4 \mathrm{~nm}$ \\
\hline Channel doping concentration & $10^{17} \mathrm{~cm}^{-3}$ \\
\hline Source/Drain doping concentration for NMOS & $2.5 \times 10^{19} \mathrm{~cm}^{-3}$ \\
\hline Source/Drain doping concentration for PMOS & $2.9 \times 10^{19} \mathrm{~cm}^{-3}$ \\
\hline Supply voltage $\left(V_{\mathrm{DD}}\right)$ & $0.9 \mathrm{~V}$ \\
\hline Gate material & Tungsten \\
\hline Gate oxide material & $\mathrm{HfO}_{2}$ \\
\hline
\end{tabular}

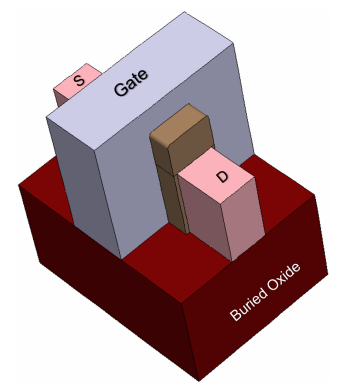

(a)

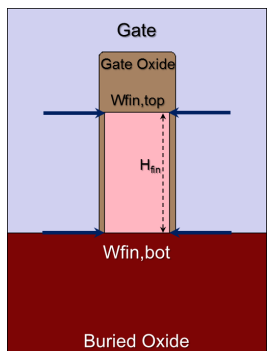

(c)

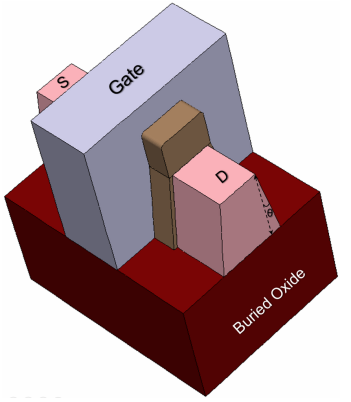

(b)

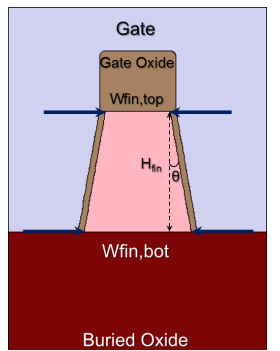

(d)
Fig. 2. Schematic representation of double-gate SOI FinFET (a) rectangular FinFET, (b) trapezoidal FinFET in 3D view, (c) rectangular FinFET, (d) trapezoidal FinFET in 2D crosssectional view.

value (e.g., fin width), the lower level of the fin becomes larger as the angle of the sidewall $(\theta)$ increases for generating trapezoidal FinFETs. While keeping the top fin width WFin,top constant, the inclination angle $(\theta)$ of the bottom fin width WFin, bot in Fig. 2(d) is a variable in the cross section geometry in this study. The 20-nm FinFET models [14] are used to calibrate the FinFET parameters. The parameters are based on the level 105.03 of BSIM compact models [15] for bulk FinFET devices. Model data is obtained using HSPICE simulation [16]. A comparison of the calibrated $I_{\mathrm{D}^{-}} V_{\mathrm{GS}}$ curve between the

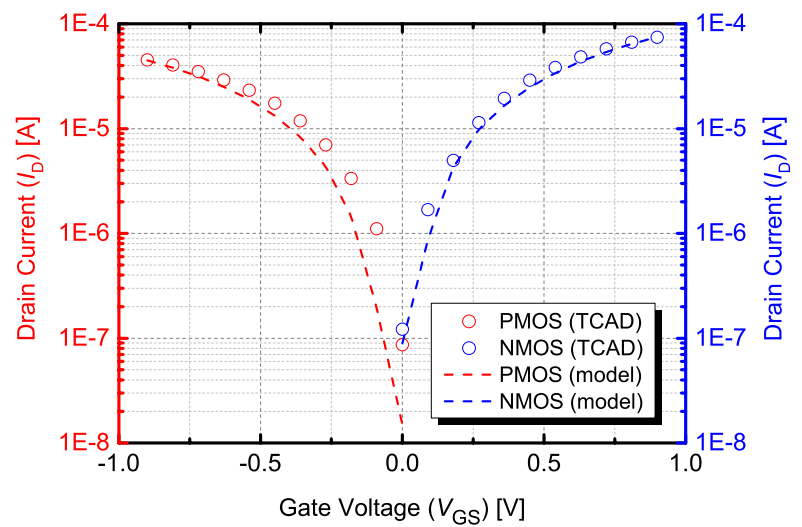

Fig. 3. Calibrated $I_{\mathrm{D}}-V_{\mathrm{GS}}$ curve. The data of a 20-nm FinFET model [14] is used to calibrate parameters for the TCAD simulations.

TCAD simulation and the models is shown in Fig. 3, and good agreement is obtained for the TCAD structure. In reality, the post-lithography process of the photoresist (PR) results in a wider PR at the bottom with respect to the top, yielding a trapezoidal shape [17]. The nonideal geometry of the PR is eventually transferred to the fin during the etching process. If we have a constant top fin width and increased sidewall angle, we have a larger effective channel area of the fin, and this in turn causes higher conduction currents than a nominal rectangular area.

The electron density profiles in the on- and off-states are shown in Fig. 4 to compare the electrical properties in both cases of the trapezoidal cross section. Fig. 4(a)-(c) show the profiles for the on-state, and (d)-(f) show the off-stage profiles. Fig. 4(b) and (e) are the cases with the same area for the on-state and off-state, respectively. Plots of $I_{\text {on }} \& I_{\text {off }}$ vs. angle in both cases are shown in (g) and (h). Fig. 5(a) shows the currents for a fixed-top width, and (b) shows the same-area case. As shown in the plots, the case of the same area for both states Fig. 5(b) produces lower conducting currents with higher leakage currents as the angle increases. However, a FinFET with a fixed top width Fig. 5(a) produces higher conducting currents with increased leakage current. Therefore, it is highly probable that the originally desired fin width ( $\left.W_{\text {fin }}\right)$ is assumed to be WFin,top defined by the photolithography, whereas the bottom of the fin (WFin,bot) has a larger thickness because of the technological limitations of the fin definition process (imperfect anisotropic etch) for devices with improved 


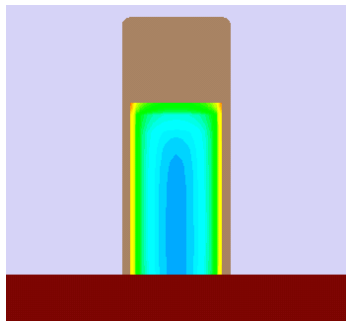

(a)

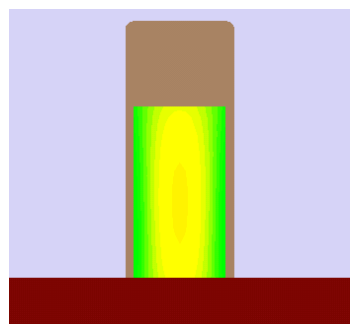

(d)

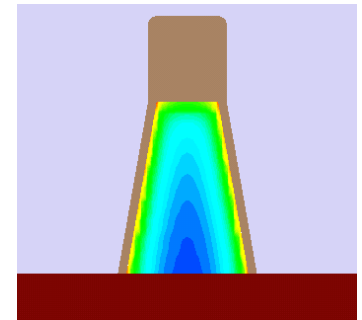

(b)

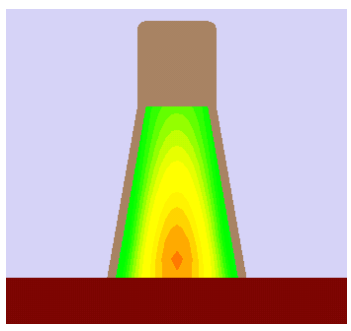

(e)

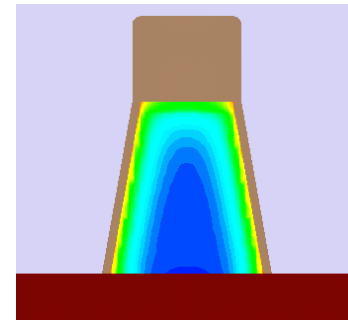

(c)

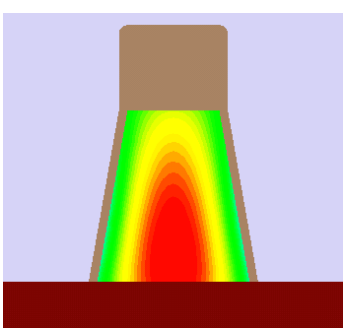

(f)
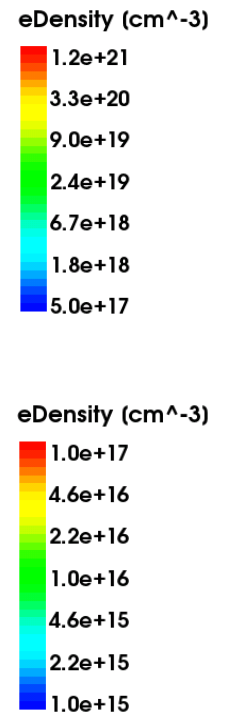

$.0 e+15$

Fig. 4. Calibrated Electron density profiles of the FinFET (a)-(c) are for the on-state, (d)-(f) are for the off-state, (a), (d) are the nominal rectangular FinFETs, (b), (e) are the on- and off-current profiles for the case with the same area for both states, (c), (f) are for the fixed Fin_ $W_{\text {top }}$ structures with a $10^{\circ}$ angle. Note the higher current density in the outer fin region in the on-state but in the center in the off-state.

driving strength. As a result, trapezoidal FinFET structures with fixed tops and varying bottom widths are used in this study. Thus, the bottom width of the fin and the length of the fin sidewall are given by

$$
\begin{aligned}
& \text { WFin, } \text { bot }=\text { WFin, top }+2 \cdot H_{\text {fin }} \cdot \tan \theta \\
& \text { Fin }_{\text {sidewall }}=H_{\text {fin }} \cdot \frac{1}{\cos \theta}
\end{aligned}
$$

A physics-based 3D TCAD simulation is used to analyze the impact of the trapezoidal fin shape in a typical $20-\mathrm{nm}$ process. In the TCAD device simulation, the following models are used to consider the physical characteristics of the narrow-fin FinFET: the dopinginduced bandgap narrowing model by Slotboom [18] and Philips unified mobility model [19], high-field mobility saturation [20], Lombardi mobility [21] to consider high$\mathrm{k}$ degradation, and a thin-layer mobility model for accurate prediction of the mobility of the FinFET.

\section{IMPACT OF ANGLE VARIATION}

The current compact model for a FinFET for a circuit simulation cannot handle the impacts of a trapezoidal fin shape. In other words, the inclination angle of the fin sidewall cannot be used as the input variable of the circuit simulator directly [14]. Thus, the properties of the trapezoidal FinFET can be analyzed in the TCAD simulations, and the impact on the electrical characteristics such as transient and $\mathrm{AC}$ behavior is investigated in mixed-mode simulations.

As mentioned in the previous section and shown in Fig. 5(a), the effective total channel width $\left(\approx 2 \times F_{i n}\right.$ sidewall $)$ increases when the inclination angle of the fin height increases. Widening the angle of the fin height creates the possibility of increasing the channel surface area, which leads to a higher on-current. On the other hand, the gate controllability in the channel becomes worse as the angle increases because of a thicker channel width between two side gates around the bottom of the fin, which becomes a penalty for the leakage current as shown in Fig. 4(f) and 5(a). Moreover, increasing the angle has a directly increases the gate capacitance because the gate capacitance is proportional to the channel area. In fact, in the case of a rectangular sidewall FinFET, the fin height is optimized for performance between the drive current and capacitance for the same reason as the channel area dependency of the gate capacitance [22]. Capacitance-voltage (CV) characteristics of trapezoidal FinFETs are investigated and are shown in Fig. 6. As can be observed, the total gate capacitance increases as the angle of the fin height increases. The 


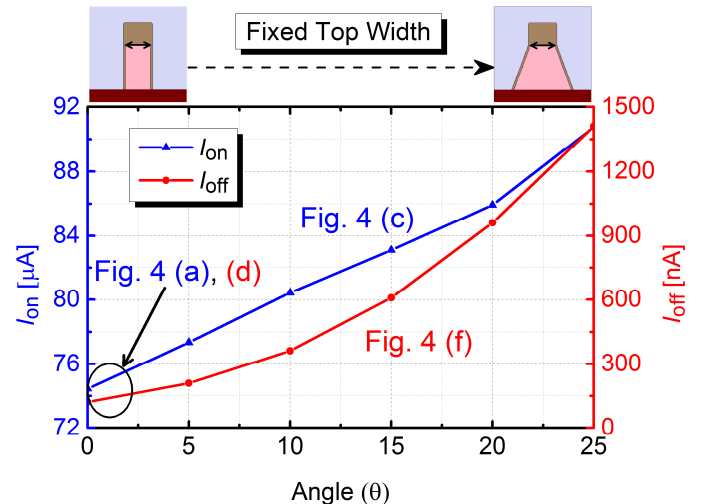

(a)

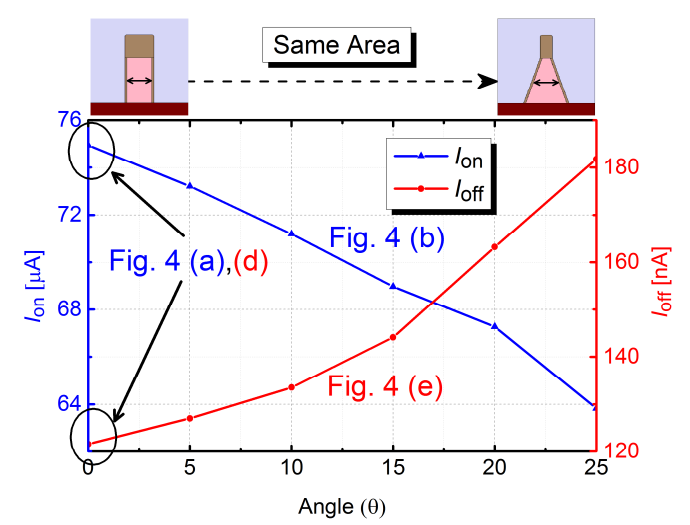

(b)

Fig. 5. $I_{\text {on }} \& I_{\text {off }}$ according to the angle variation of fixed-top and same-area cases are shown in (a) and (b), respectively.

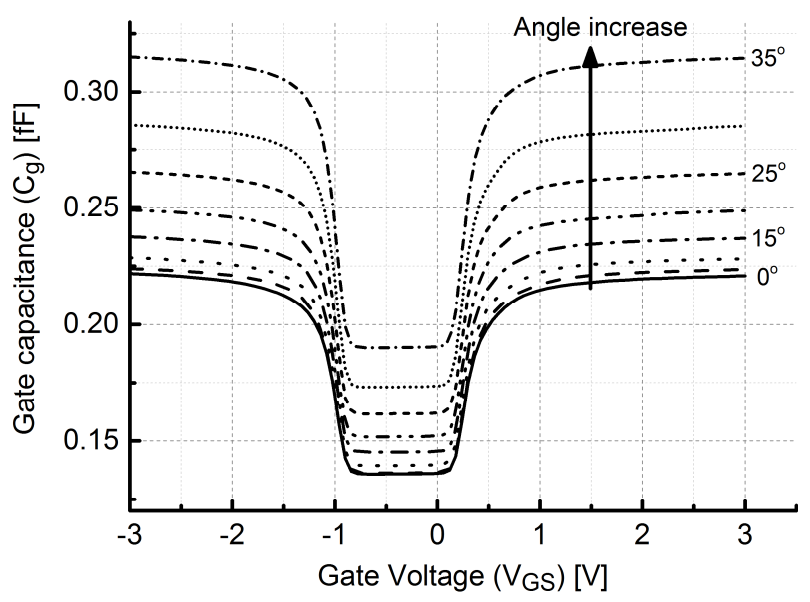

Fig. 6. A simulated capacitance-voltage curve of the FinFETs with varying angle. Frequency is set to be $1 \mathrm{MHz}$ for simulation. The curves move upward as the angle of the fin increases.

drain current (i.e., on-current or $I_{\text {on }}$ ) and gate capacitance according to the inclination angle variation are shown in Fig. 7. The percentage increases are summarized in the table inside the figure. A $0^{\circ}$ angle implies a perfectly

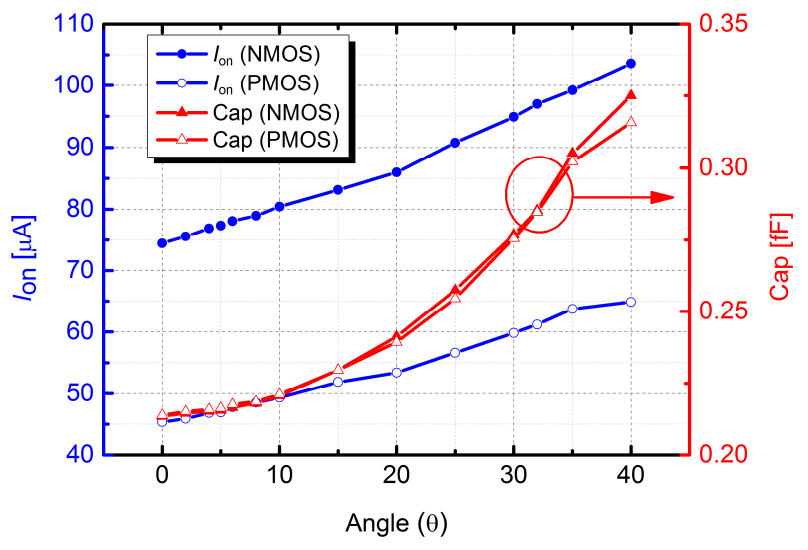

Fig. 7. The drain current in red (left y-axis) and gate capacitance in blue (right y-axis) of FinFETs according to the inclination angle variation.

Table 2. Percentage increase in drive current and gate capacitance associated with Fig. 7

\begin{tabular}{|c|c|c|c|c|}
\hline & $I_{\text {on }}(\mathrm{n})$ & $I_{\text {on }}(\mathrm{p})$ & $C_{\mathrm{g}}(\mathrm{n})$ & $C_{\mathrm{g}}(\mathrm{p})$ \\
\hline $0^{\circ}$ to $20^{\circ}$ & $12 \%$ & $14 \%$ & $8 \%$ & $7 \%$ \\
\hline $20^{\circ}$ to $40^{\circ}$ & $25 \%$ & $25 \%$ & $42 \%$ & $37 \%$ \\
\hline $0^{\circ}$ to $40^{\circ}$ & $39 \%$ & $43 \%$ & $52 \%$ & $48 \%$ \\
\hline
\end{tabular}

rectangular FinFET. Note that for an angle up to approximately $20^{\circ}$, the percentage increase in $I_{\text {on }}$ and the capacitance are relatively small (e.g., up to $14 \%$ for $I_{\text {on }}$ and up to $8 \%$ for the capacitance). However, for angles greater than $20^{\circ}$, the capacitance increases significantly more than $I_{\text {on }}$ (e.g., $42 \%$ versus $25 \%$ in an n-FinFET). This result indicates that there are certain ranges of the trapezoidal angles for better dynamic performance than the nominal FinFET.

The delay of the transistor depends on the relationship between the driving current strength $(I)$, the gate capacitance $(C)$, and the supplied voltage of operation $(V)$. The intrinsic delay $(\tau)$ of a conventional transistor is given by

$$
\tau=\frac{C \cdot V}{I}
$$

Thus, both $I_{\text {on }}$ and the capacitance affect the dynamic performance of the circuits. In addition, there is a tradeoff between the two characteristics in the trapezoidal FinFET. As the inclination angle is varied in the fin sidewall according to the trapezoidal FinFET shape, the delay of the FinFET increases or decreases according to the relative percentage changes in the 
capacitance and drive current. Therefore, when the angle exceeds $20^{\circ}$, the benefit of having a larger current in the delay disappears because of the greater capacitance increase. In the following section, several benchmark circuits are exploited in the mixed-mode simulations to investigate the impact of the trapezoidal angle on the circuit performance.

\section{Impact on Circuit Performance}

As explained in the previous section, there is a tradeoff between the driving current and the gate capacitance (and leakage current as well) as the trapezoidal angle increases. In other words, when the bottom of the fin width increases (i.e., wider inclination angle), the driving current increases, but the leakage current (and dynamic power) and gate capacitance increase simultaneously. However, their relative impacts on the dynamic characteristic of the circuit are different in various performance metrics.

In order to verify the impact of the trapezoidal FinFET on the circuit level quantitatively, four circuit examples

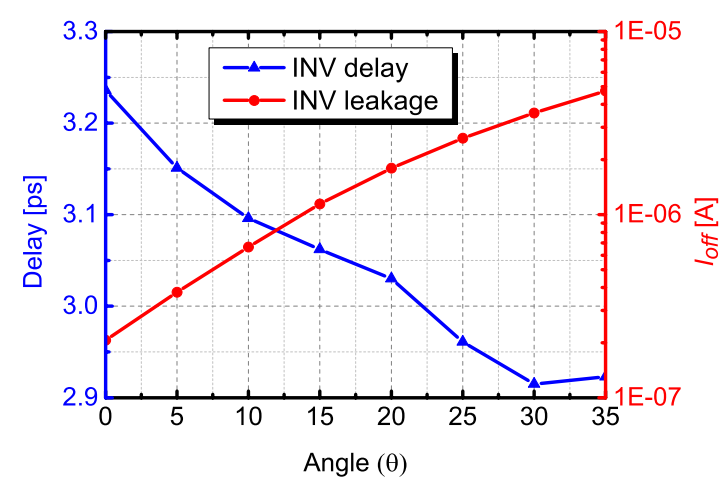

(a)

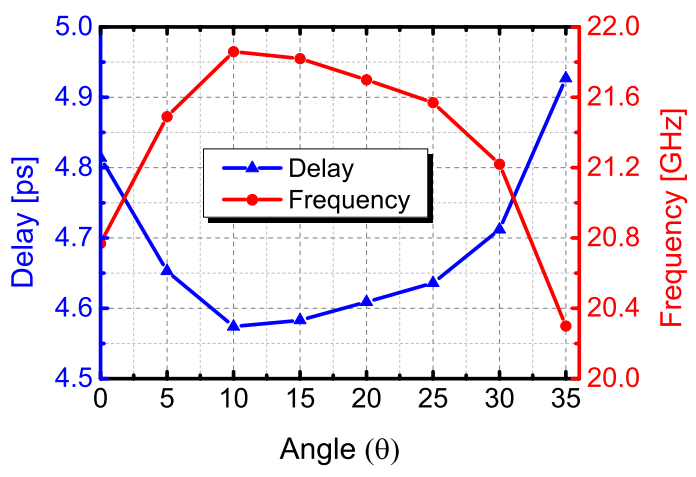

(c)

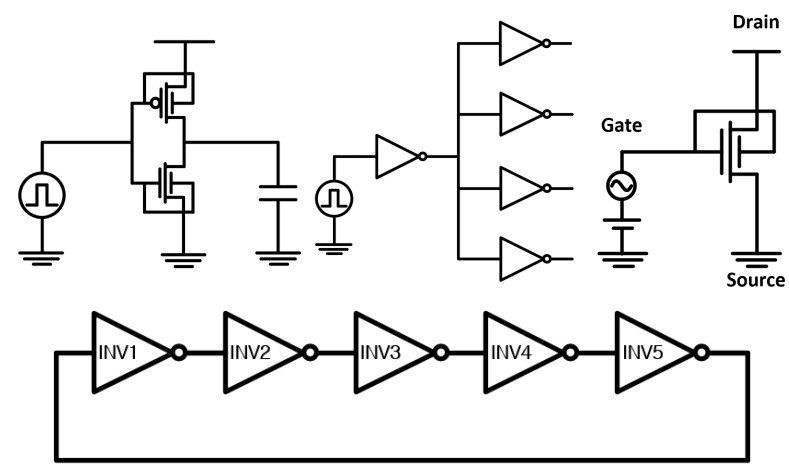

Fig. 8. Schematics used in Sentaurus device mixed-mode simulations (a) Inverter with fixed loading capacitance, (b) FO4 inverter, (c) NMOS for AC simulation, (d) 5-stage ring oscillators.

are considered in the Sentaurus Device mixed-mode simulations as performance metrics, as depicted in Fig. 8: a single inverter with a fixed loading cap, a fan-out 4 inverter, and a five-stage ring oscillator. In addition, the cut-off frequency $\left(f_{\mathrm{T}}\right)$ is measured in the AC simulation to investigate the impact of the trapezoidal FinFET on analog applications. The simulation results for the delays of the FO4 inverter and five-stage RO are shown in Fig.

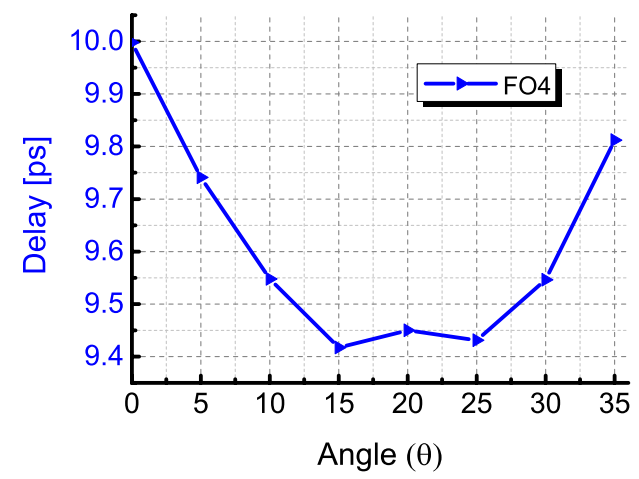

(b)

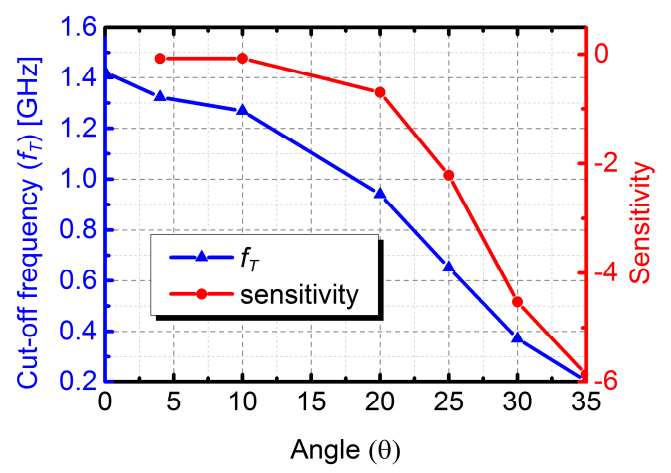

(d)

Fig. 9. Impact on the performance according to inclination angle variation. (a) Inverter with fixed loading capacitance, (b) FO4 inverter, (c) ring oscillators, and (d) cut-off frequency. 
9. As expected, the propagation delays of the trapezoidal FinFETs always decrease (or faster) as the angle increases in the fixed loading capacitor case in Fig. 9(a) with penalty on the leakage power. In this study, the increased angle of the fin enables a larger area for the conduction channel because WFin,bot is assumed to have a larger thickness than the nominal thickness, and this, in turn, causes higher conduction currents. Therefore, the circuits in the trapezoidal FinFET with a fixed loading capacitor have a shorter delay than the nominal delay (i.e., $0^{\circ}$ ).

On the other hand, interesting results are observed for the FO4 inverter (Fig. 9(b)) and five-stage RO (Fig. 9(c)). The delays decrease up to some angle (e.g., $15^{\circ}$ for the FO4 inverter and $10^{\circ}$ for the RO) and increase for larger angles. In this case, as mentioned in Section III, the penalty associated with an increase in the capacitance becomes larger for significantly larger angles and the ratio of the capacitance increase is similar to the ratio of driving current increase up to $20^{\circ}$. The impact on the oscillating frequency variations at the output node (INV3) produced by the angle variations is shown in Fig. 9(c). As shown, the RO generates a higher frequency as the angle increases, and the maximum frequency occurs at $10^{\circ}$ for the trapezoidal FinFET. However, the increased gate capacitance dominates from $20^{\circ}$ by reducing the oscillating frequency than the optimum angle (e.g., $10^{\circ}$ or $15^{\circ}$ ). The transient response of the RO in Fig. 10 clearly shows the behavior of the frequency according to the angle.

As the figure of merit for RF performance, $f_{\mathrm{T}}$ is observed to understand the device behavior for analog applications. $f_{\mathrm{T}}$ is extracted when the current gain is unity at the proper bias for trapezoidal NMOS FinFETs with various angles. As shown in Fig. 9(d), a smaller cut-off frequency is obtained as the trapezoidal angle of the FinFET increases owing to the larger capacitance effect, as observed from the following equation:

$$
f_{T} \approx \frac{g_{m}}{2 \pi\left(C_{g s}+C_{g d}\right)}
$$

The gate-source $\left(C_{\mathrm{gg}}\right)$ and gate-drain $\left(C_{\mathrm{gd}}\right)$ capacitance components are proportional to the effective width of the device, and the effective width increases as the angle

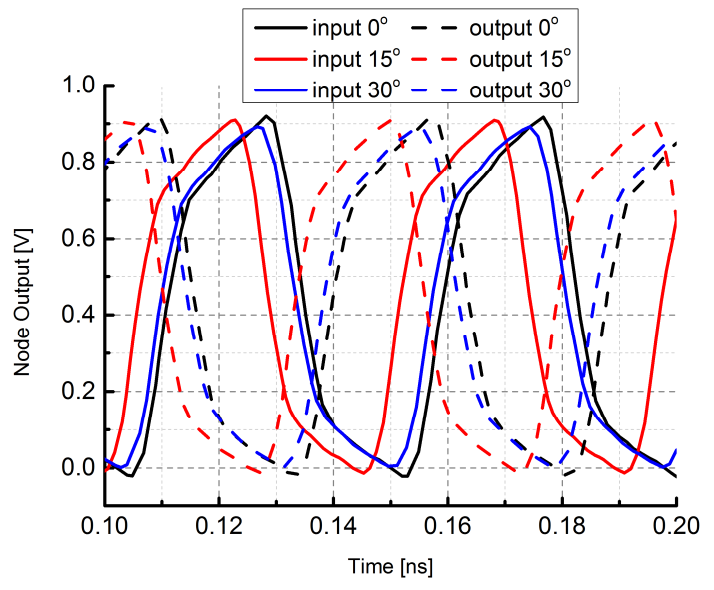

Fig. 10. The node output (inv3) variations produced by the angle variations. The output is the third stage node output in the 5-stage ring oscillator.

increases. Thus, even though a larger transconductance, $g_{\mathrm{m}}$, is realized by the trapezoidal FinFET, the higher capacitance degrades $f_{\mathrm{T}}$. We adopt the dimensionless derivatives of the cut-off frequency with respect to the inclination angle $(\theta)$ and their ratios as follows [23]:

$$
f_{T_{-} \theta}=\frac{\theta}{f_{T}} \frac{\partial f_{T}}{\partial \theta}
$$

The relative sensitivity according to the angle variation, $f_{\mathrm{T}_{-} \theta}$, is shown in Fig. 9(d). As shown, $f_{\mathrm{T}}$ is weakly sensitive to the angle up to $20^{\circ}$ but degrades significantly above $20^{\circ}$. This result agrees with the dominant effect of the capacitance for larger angles (e.g., $\theta \geq 20^{\circ}$ )

\section{CONCLUSIONS}

In this study, the performance impact of the trapezoidal fin shape of a double-gate FinFET is analyzed with numerous TCAD simulations. Several performance metrics are evaluated to investigate the impact of the trapezoidal fin shape on the circuit operation. The TCAD simulations show that the driving capability improves, and the gate capacitance increases as the bottom fin width of the trapezoidal fin increases. The trapezoidal FinFET with a fixed loading capacitance validates the increase in the driving current. However, the increase in the gate capacitance nullifies the benefit of the current increase due to the trapezoidal fin a larger 
angle. The FO4 inverter and RO delay results indicate that careful optimization of the trapezoidal angle can increase the speed of the circuit because the ratios of the current and capacitance have different impacts depending on the trapezoidal angle. $f_{\mathrm{T}}$ is observed to understand the device behavior at high frequencies. The impact of the trapezoidal FinFET on the cut-off frequency correlates well with the observations from the driving current and capacitance. As a result, careful control of the trapezoidal angle in the FinFET devices will be beneficial to circuit performance.

\section{ACKNOWLEDGMENT}

This research was supported by Basic Science Research Program through the National Research Foundation of Korea (NRF) funded by the Ministry of Education (NRF-2014R1A1A2057715). EDA tools were partially supported by IDEC at KAIST.

\section{REFERENCES}

[1] Y. Taur and T. H. Ning, Modern VLSI devices. Cambridge, UK: Cambridge Univ. Press, 2009.

[2] R. Huang et al., "Challenges of $22 \mathrm{~nm}$ and beyond CMOS technology," Science in China Series F: Information Sciences, vol. 52, no. 9, pp. 1491-1533, 2009.

[3] M. Bohr, "The evolution of scaling from the homogeneous era to the heterogeneous era," in International Electron Devices Meeting (IEDM), 2011, pp. 1-1.

[4] E. J. Nowak et al., "Turning silicon on its edge double gate CMOS/FinFET technology," Circuits and Devices Magazine, IEEE, vol. 20, no. 1, pp. 20-31, 2004.

[5] Intel Corporation. "3-D, 22nm: New Technology Delivers An Unprecedented Combination of Performance and Power Efficiency". [Online]. Available:http://www.intel.com/technology/archite cturesilicon/22nm/

[6] P. Clarke. "Intel's FinFETs are less fin and more triangle". [Online]. Available: http://www.eetimes.com

[7] C. Auth et al., "A 22nm high performance and lowpower CMOS technology featuring fullydepleted tri-gate transistors, self-aligned contacts and high density MIM capacitors," in VLSI Technology (VLSIT), 2012 Symposium on. IEEE, 2012, pp. 131-132.

[8] C.-H. Lin et al., "Channel doping impact on finfets for 22nm and beyond," in VLSI Technology (VLSIT), 2012 Symposium on, June 2012, pp. 15-16.

[9] J. P. Duarte, N. Paydavosi, S. Venugopalan, A. Sachid, and H. Chenming, "Unified FinFET compact model: Modelling Trapezoidal Triple-Gate FinFETs," in Simulation of Semiconductor Processes and Devices (SISPAD), 2013 International Conference on, 2013, pp. 135-138.

[10] X. Wu, P. C. Chan, and M. Chan, "Impacts of nonrectangular fin cross section on the electrical characteristics of FinFET," Electron Devices, IEEE Transactions on, vol. 52, no. 1, pp. 63-68, 2005.

[11] R. Giacomini and J. A. A. Martino, "Non-Vertical Sidewall Angle Influence on Triple-Gate FinFETs Corner Effects," ECS Transactions, vol. 6, no. 4, pp. 381-386, 2007.

[12] W. Kehuey, D. Wei-Wen, and C. Meng-Hsueh, "Performance advantage and energy saving of triangular-shaped FinFETs," in Simulation of Semiconductor Processes and Devices (SISPAD), 2013 International Conference on, 2013, pp. 143146.

[13] Sentaurus TCAD User Guide, Ver. H-2013.03. [Online]. Available: http://www.synopsys.com

[14] S. Sinha, G. Yeric, V. Chandra, B. Cline, and Y. Cao, "Exploring sub-20nm FinFET design with predictive technology models," in Proceedings of the 49th Annual Design Automation Conference. ACM, 2012, pp. 283-288.

[15] M. V. Dunga, C.-H. Lin, D. D. Lu, W. Xiong, C. Cleavelin, P. Patruno, J.-R. Hwang, F.-L. Yang, A. M. Niknejad, and C. Hu, "BSIM-MG: A versatile multi-gate FET model for mixed-signal design," in VLSI Technology, 2007 IEEE Symposium on. IEEE, 2007, pp. 60-61.

[16] HSPICE, Ver. H-2013.03. [Online]. Available: http://www.synopsys.com

[17] D. Zhang, S. Rauf, and T. Sparks, "Modeling of photoresist erosion in plasma etching processes," Plasma Science, IEEE Transactions on, vol. 30, no. 1, pp. 114-115, 2002.

[18] J. Slotboom, "The pn-product in silicon," SolidState Electronics, vol. 20, no. 4, pp. 279-283, 1977. 
[19] D. Klaassen, “A unified mobility model for device simulation-I. Model equations and concentration dependence," Solid-State Electronics, vol. 35, no. 7, pp. 953-959, 1992.

[20] D. Sinitsky, F. Assaderaghi, C. Hu, and J. Bokor, "High field hole velocity and velocity overshoot in silicon inversion layers," Electron Device Letters, IEEE, vol. 18, no. 2, pp. 54-56, 1997.

[21] C. Lombardi, S. Manzini, A. Saporito, and M. Vanzi, "A physically based mobility model for numerical simulation of nonplanar devices," Computer-Aided Design of Integrated Circuits and Systems, IEEE Transactions on, vol. 7, no. 11, pp. 1164-1171, 1988.

[22] M.-h. Chi, "Challenges in Manufacturing FinFET at 20nm node and beyond," Technology Development, Global Foundries, Malta, NY, USA, 2012.

[23] V. Zyuban and P. Strenski, "Unified methodology for resolving powerperformance tradeoffs at the microarchitectural and circuit levels," in Proceedings of the 2002 international symposium on Low power electronics and design. ACM, 2002, pp. 166-171.

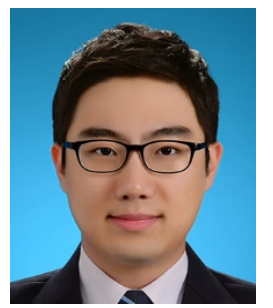

Myunghwan Ryu received B.S. degree in electrical engineering and computer science from Kyungpook National University, Daegu, Korea, in 2010, and M.S. degree in electrical and computer engineering from the Ulsan National Institute of Science and Technology (UNIST), Ulsan, Korea, in 2012, respectively, where he is currently working toward a Ph.D. degree in electrical engineering. His current research interests include TCAD-based compact modeling development for nanoscale CMOS/FinFETs, and Design Technology CoOptimization methodologies, and design, simulation, modeling for variability of various emerging devices.

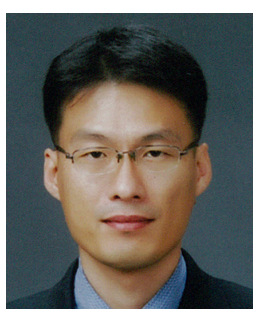

Youngmin Kim received the B.S. degree in electrical engineering from the Yonsei University, Seoul, Korea, in 1999, and the M.S. and Ph.D. degrees in electrical engineering from the university of Michigan, Ann Arbor, in 2003 and 2007, respectively. He has held a senior engineer position in the Qualcomm, San Diego, CA. He is currently an Assistant Professor in Kwangwoon University, Seoul, South Korea. Prior to joining Kwangwoon University, he was with the school of electrical engineering and computer engineering at the Ulsan National Institute of Science and Technology (UNIST), Ulsan, South Korea. His research interests include variability-aware design methodologies, design for manufacturability, and design and technology cooptimization methodologies, and low-power and 3D IC designs. 